\title{
La empleabilidad de los universitarios en Europa. Contribuciones de la educación no formal
}

\section{The employability of graduates in Europe. Contributions of non-formal education}

\author{
Jesús García-Álvarez*, Ana Vázquez-Rodríguez*, Ígor Mella-Núñez* \\ *Grupo de Investigación Esculca-Rede RIES, Universidade de Santiago de Compostela
}

\begin{abstract}
Resumen
El objetivo principal de este trabajo es analizar la incidencia de la educación no formal en la empleabilidad de los graduados. Para ello, realizamos una revisión de investigaciones que, específicamente, seleccionaron variables ligadas a la participación de los universitarios en actividades de educación no formal y su contribución al desarrollo de competencias de potencial empleabilidad. Los resultados ponen el acento en la necesidad de que el sistema universitario reconsidere la importancia de su "tercera misión" para dar una mayor visibilidad a sus actividades. Esto implica asegurar que las prioridades de los agentes sociales/económicos estén presentes en sus actividades. Palabras clave: graduados, empleabilidad, educación no formal, educación superior, mercado laboral.
\end{abstract}

\section{Abstract}

The main objective of this paper is to identify and analyse the impact of non-formal education on the employability of graduates. Using qualitative methodologies, we review major studies and reports that took into account, specifically, variables associated to the participation of graduates in non-formal education and their contribution to the development of career building skills. The results indicate the need for universities to reconsider the importance of their "third mission" to give it more salience in their activities. This would entail, in particular, making sure that social and economic actors and priorities are present in their activities.

Keywords: graduates, employability, non-formal education, higher education, labour market.

\section{Introducción}

Es evidente que la educación superior ha de ser el motor de la nueva sociedad del conocimiento. La formación de graduados, así como la transferencia del conocimiento obtenido por las universidades constituye un elemento clave de desarrollo. El papel del conocimiento en el cambio del modelo social y económico es fundamental y la universidad se proyecta como el agente principal de producción y transferencia del mismo.

El nuevo modelo de educación superior destaca por la importancia concedida a las competencias profesionales y la empleabilidad como elemento de cambio. El sistema ha de articularse en torno a estos dos ejes. La preocupación por la empleabilidad es un problema, tanto para la comunidad educativa y científica, como para las instituciones políticas y sociales. Concretamente, la Estrategia Europa 2020, fija entre sus objetivos adecuar los planes de estudio para que satisfagan las necesidades de las personas, del mercado de trabajo y de las carreras del futuro; formar a los investigadores a preparar el terreno para las industrias del mañana; dar más oportunidades a los estudiantes para que adquieran competencias vinculadas al empleo, así como potenciar los lazos entre la enseñanza, la investigación y la empresa (Comisión Europea, 2013).

Los estudiantes esperan que las universidades los apoyen, no solo en su desarrollo intelectual y académico, sino también estableciendo una plataforma para el empleo y el desarrollo de sus carreras. Ellos han de conocer y comprender las tendencias que están en juego para poder tomar las decisiones correctas. Las estrategias que las universidades requieren para ello, deben realizarse con la voluntad de implicar a los agentes sociales, políticos y económicos de su entorno.

Si lo que buscamos es que nuestras universidades sean más competitivas, deberán potenciar, además de la docencia, la colaboración con el sector productivo y la administración. En relación con ello, el desarrollo de acciones específicas enmarcadas en el sistema de educación no formal, constituye una buena oportunidad de conectar la formación universitaria con el mundo empresarial, potenciando la empleabilidad de los graduados.

En este sentido, entendemos por educación no formal todas aquellas actividades, estrategias o acciones que no están contempladas en la legislación estatal sobre educación; es decir, su responsabilidad no recae directamente en los ordenamientos jurídicos del Estado. Los cursos, seminarios y jornadas serían un ejemplo de educación no formal, pues si bien se llevan a cabo en instituciones educativas oficiales como son las propias universidades, su marco legal no viene definido por la legislación ministerial sino por la universidad misma. Tener en cuenta cualquier otra 
variable puede llevar a confusión, pues la educación formal o no formal aceptan múltiples aspectos comunes como puede ser el espacial, el profesional, el sistemático o por el contrario la flexibilidad, la racionalidad, la planificación y lo evaluativo (Colom, 2005).

De este modo, tomando como referencia la dificultad a la que actualmente se enfrentan la mayoría de los graduados universitarios, es decir, considerando el cambio producido en la efectividad del sistema universitario como vía de acceso al empleo, identificamos un conjunto de estrategias que intervienen directamente sobre la cuestión, especificando cada una de ellas en acciones más concretas.

\section{Método}

Empleando una metodología de corte cualitativo, hemos analizado una serie de investigaciones ligadas a la importancia que determinadas actividades, enmarcadas en el sistema de educación no formal, tienen en el acceso al empleo de los titulados universitarios. Concretamente, a partir de un enfoque interpretativo, y con una clara orientación ideográfica, identificamos un conjunto de estrategias de potencial empleabilidad para los universitarios europeos.

\section{Resultados}

La institución universitaria se enfrenta hoy al desafío de generar iniciativas, colectivas e individuales, dirigidas a fomentar la empleabilidad de sus graduados. La creación de un espacio de relación como resultado de la participación de las tres grandes esferas (universidad, empresa y administración pública) constituye el centro articulador de proyectos capaces de intervenir sobre la realidad sociolaboral que atraviesa la población más joven. Solamente cuando las universidades se apoyen en la empresa serán capaces de formar a un colectivo preparado para hacer frente a las condiciones del nuevo mercado de trabajo. Y sólo podrán, las empresas, dar respuesta a las necesidades productivas impuestas por la globalización del mercado si se apoyan en las innovaciones que generan las instituciones de educación superior.

Para ello, la universidad deberá replantear su actual estructura pues la lentitud que caracteriza el sistema de educación formal provoca que, desde el momento en el que se localizan determinadas necesidades, y hasta que se activan las estrategias oportunas, han aparecido nuevos cambios para los que aún no está preparada. Como ejemplo, puede observarse como en la práctica, la formación destinada a dar respuesta a profesiones emergentes, se realiza, principalmente, a través del sistema de educación no formal y transcurren años hasta que la educación formal implanta nuevas titulaciones y especialidades (Esteve, Vera, y Aznar, 1991).

El valor de la universidad radica en tanto organización colectiva cuyos miembros, con capacidad de interpretación, analizan el significado del entorno en el que se encuentran inmersos y ofrecen, a quienes forman, los elementos imprescindibles para integrarse, no solo adecuadamente, sino con los conocimientos y competencias necesarias para identificar las dificultades de cada etapa. La colaboración con los demás sistemas sociales le proporcionará credibilidad en el mercado laboral, presentándose como eje articulador de nuevas políticas socioeducativas.

Es cierto que la universidad ha progresado de un modo notable en los últimos años, aun cuando el Espacio Europeo de Educación Superior (EEES) ha supuesto la aplicación de reformas que finalmente no se han consolidado. En suma, los recortes en gasto público, consecuencia de la recesión, no han permitido alcanzar muchos de los logros planteados inicialmente $\mathrm{y}$, otros muchos, los ha puesto en riesgo. Sin embargo, es importante continuar progresando, examinando las ineficiencias y activando vías alternativas que constituyan una mejora del sistema universitario, el cual ha finalizado su proceso de adaptación al EEES (Martín-González, 2013).

Ante el reto de transformar rápidamente la sociedad tradicional en una nueva sociedad de la información y del conocimiento, el sistema universitario ha de aprovechar las enormes potencialidades del sector productivo (Casas, 2005). En este sentido, se advierte la importancia del factor conocimiento, su producción, aplicación y utilización en los distintos ámbitos de trabajo (Albornoz, 2002). En consecuencia, el instrumento principal para asumir estas transformaciones y adaptarse a ellas es la educación y, dentro de la misma, la universidad ocupa un lugar especial (Castells, 2005).

En base a ello, identificamos la primera de las estrategias que la universidad ha de poner en marcha y que se desarrollaría en paralelo al sistema de educación formal. Esta, que ya habría sido abordada por García-Álvarez y Fernández-Salinero (2016), se refiere a la implementación de un proceso de gestión del conocimiento que busca promover toda una serie de mecanismos de los que pueda hacer uso el egresado y que le proporcionarán los recursos y herramientas necesarias para enfrentarse a las dificultades del mercado de trabajo.

Tal misión, implica redefinir los objetivos de la universidad y enfocarlos hacia la construcción personal del conocimiento y no tanto a la simple adquisición del mismo, facilitando la discriminación coherente en la selección y filtración de la información más relevante, la creatividad para gestionar situaciones de incertidumbre, la innovación orientada al proceso y producto, la confianza en las posibilidades de aprendizaje de cada individuo, así como la consideración de los centros de formación como redes de conocimiento capaces de facilitar procesos de coproducción e interacción (Fernández-Salinero, 2012).

En este sentido, la propuesta se basa en convertir el conocimiento tácito en conocimiento explicito, de modo que pueda ser documentado y almacenado con la finalidad de utilizarlo en el momento oportuno. Ello 
exige, por parte de la universidad, la promoción de habilidades de desarrollo profesional relacionadas con la identificación y uso de información sobre una profesión determinada que, sin duda, contribuirán a establecer expectativas más coherentes con la situación del mercado laboral (Bridgstock, 2009). Para ello, pueden emplearse herramientas como las bases de datos, intranet, revistas, manuales, etc. que permiten almacenar el conocimiento extraído.

La idea es crear un entorno donde el conocimiento y la información disponibles en una determinada organización sean accesibles y puedan utilizarse para estimular la actualización y mejorar la toma de decisiones. Así, la gestión del conocimiento, se entiende como la disciplina adecuada para intervenir sobre las nuevas necesidades de la educación superior, tanto en la organización de la institución educativa como en sus diferentes funciones (Encina, 2008). La clave pasa por crear una cultura en la que la información y el conocimiento se gestionen y usen eficaz y eficientemente.

Esto nos sitúa ante la segunda estrategia a desarrollar en el entorno universitario, fomentar el acceso del alumnado a los sistemas de orientación profesional, implicando para ello al sector socioeconómico.

Fomentar el acceso a la orientación y la información tanto en la etapa educativa como profesional de un modo continuo que permita a estudiantes y profesionales, en cualquier momento de sus vidas, identificar sus capacidades, competencias e intereses, así como adoptar decisiones educativas, formativas y ocupacionales (Consejo de Europa, 2004, 2008). La orientación ha de considerar un amplio espectro de actividades individuales y colectivas relacionadas con el intercambio de información, el asesoramiento, la evaluación de competencias, el apoyo, y el desarrollo de capacidades para la toma de decisiones y gestión de la carrera, entre otras; reconociéndola como una de las políticas activas que presentan un mayor impacto sobre la formación y el empleo de los titulados superiores. Así, universidades y organizaciones empresariales deberían replantear su estructura situando la orientación académico-profesional como un importante pilar en su actividad. Esto mejoraría en las universidades el conocimiento que el alumno posee sobre sus posibilidades en el mercado laboral y le proporcionaría las herramientas para enfrentarse al mismo. En las empresas, ajustaría los perfiles profesionales a las ocupaciones, optimizando procesos formativos más coherentes y aumentando la eficiencia de la organización.

Se halla que la orientación profesional mejora el nivel de empleabilidad de los egresados, pues contribuye positivamente al acceso al empleo de los egresados en general y al desarrollo de competencias transversales y de manejo de los recursos y las herramientas de un modo particular y especialmente significativo. Existe una clara correlación entre los egresados que reciben orientación profesional por competencias y una coyuntura laboral satisfactoria en los mismos. Al igual que existe una correlación marcada entre los egresados que han recibido orientación profesional por competencias y la propia percepción que tienen de su nivel de empleabilidad, considerándose ellos mismos más empleables al haber recibido dicha orientación (Rodríguez Martínez, 2012.

Teniendo en cuenta la índole de las estrategias que tanto a la educación superior como al sector productivo se le exigen, no podemos descuidar el marco normativo en el que han de disponerse y, por ende, a los encargados de tomar decisiones y fijar las políticas correspondientes. Identificamos así la tercera y última de las estrategias que, aunque en sí misma no supone el desarrollo de ninguna acción educativa tanto formal como no formal, constituye un apoyo claro para consolidar las anteriores. Ella se refiere al desarrollo de una política educativa en clara conexión con la política de empleo. Ambas presentan considerables carencias en sus propuestas sobre formación profesional, por lo menos en lo que a contenidos pedagógicos y particularmente didácticos se refiere. En un estudio iniciado por Arpe, Hellmann, Kenis, Löber, \& Mühlbacher, (2017) más de mil científicos evaluaron las reformas en el ámbito de las políticas sociales entre mediados de 2014 y principios de 2016 en dimensiones como la educación y el empleo. Según el mismo, España ocuparía, en la Unión Europea de los Veintiocho, el puesto veinticinco (25) sobre políticas educativas y el puesto veintisiete (27) sobre políticas de acceso al mercado laboral, debido, en su mayoría, a la visión sectorial de sus políticas públicas que, en el mejor de los casos, habrían incrementado la distancia entre los extremos de sus polos.

Paliar esta carencia sugiere enmarcar la formación profesional en un contexto compartido de similar consideración por parte de la política educativa y de empleo. La necesaria relación entre ambas ha de basarse en principios claros y objetivos bien definidos, estableciendo rigurosos mecanismos que analicen y controlen los resultados académico-profesionales de los que en tal sistema se forman.

El carácter universal del aprendizaje y la investigación, al que obedece la internacionalización de la educación superior, no se ve fortalecido por los actuales procesos de integración política. Ha de reivindicarse la formación universitaria no sólo desde una perspectiva educativa, sino también económica, sociológica, empresarial y política, para lo que será necesario dotar a la formación de una estructura común que aúne todas sus dimensiones. Una concreción mayor de las relaciones entre política educativa y política de empleo supondrá un beneficio en la formación de los profesionales del futuro. Por un lado, estableciendo mecanismos que permitan a las empresas trasladar sus necesidades a las universidades, generando así nuevos ejes de investigación. Por otro, ajustando el aprendizaje a las exigencias reales del sector productivo, formando profesionales en un marco sólido y coherente con las demandas que se derivan de las necesidades del mercado de trabajo.

Recogemos en la siguiente tabla, a modo de resumen, todas las estrategias y acciones enunciadas hasta el momento. 
Tabla 3.

Estrategias de ajuste entre educación superior y mercado de trabajo.

\begin{tabular}{|c|c|}
\hline Acciones & Objetivo \\
\hline 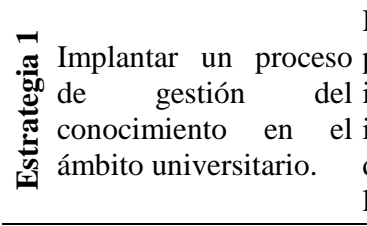 & $\begin{array}{l}\text { Desarrollar habilidades } \\
\text { profesionales relacionadas con la } \\
\text { identificación y utilización de } \\
\text { información sobre una profesión } \\
\text { determinada o sobre el mercado } \\
\text { laboral en su conjunto. }\end{array}$ \\
\hline 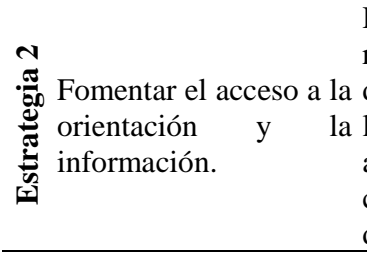 & $\begin{array}{l}\text { Desarrollar actividades } \\
\text { relacionadas con el intercambio } \\
\text { de información, el asesoramiento, } \\
\text { la evaluación de competencias, el } \\
\text { apoyo, y el desarrollo de } \\
\text { capacidades para la toma de } \\
\text { decisiones y gestión de la carrera. }\end{array}$ \\
\hline 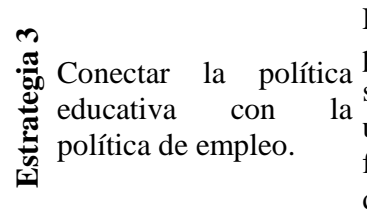 & $\begin{array}{l}\text { Establecer mecanismos que } \\
\text { permitan a las empresas trasladar } \\
\text { sus necesidades a las } \\
\text { universidades y, a estas, ajustar la } \\
\text { formación a las exigencias reales } \\
\text { del sector productivo. }\end{array}$ \\
\hline
\end{tabular}

Fuente: elaboración propia.

Es a partir de la formulación de estas estrategias donde se constituye un sistema cuya conjugación de elementos comparte similares objetivos. Universidad, administración y empresa son los encargados de que las acciones derivadas de las mismas se hagan efectivas.

\section{Discusión}

Como vemos, la universidad asume un importante papel en la iniciativa o puesta en marcha de cada una de las acciones recogidas. No obstante, no cabría esperar repercusión alguna si las demás partes implicadas no se involucran activamente en el proceso. Las universidades han sido siempre instituciones totalmente centradas en el conocimiento y su gestión, por lo tanto, constituye una oportunidad y una necesidad, en su elevada responsabilidad formadora, la preparación de escenarios para aplicar los métodos y técnicas apropiadas al contexto y a los objetivos de las organizaciones laborales (Passaillaigue y Estrada, 2016), bien desde la propia universidad, desde la empresa o desde la administración pública.

\section{Referencias}

Albornoz, M. (2002). La Universidad ante la innovación. Madrid: Fundación Santillana.

Arpe, J., Hellmann, T., Kenis, P., Löber, D., \& Mühlbacher, S. (2017). Social Policy in the EU-Reform Barometer 2016. Social Inclusion Monitor Europe. Gütersloh: Fundación Bertelsmann.

Bridstock, R. (2009). The graduate attributes we've overlooked: enhancing graduate employability through career management skills. Higher Education Research \& Development, 28(1), 31-44. http://dx.doi.org/ 10.1080/07294360802444347

Casas, M. (2005). Nueva universidad ante la sociedad del conocimiento. Revista de Universidad y Sociedad del Conocimiento, 2(2), 1-18.
Castells, M. (2005). La era de la información (Vol. 1): Economía, sociedad y cultura. La sociedad red. Madrid: Alianza.

Colom, A. (2005). Continuidad y complementariedad entre la Educación formal y no formal. Revista de Educación, 338, 9-22.

Comisión Europea. (2013). Europa 2020: la estrategia europea de crecimiento. Luxemburgo: Oficina de Publicaciones de la Unión Europea.

Consejo de Europa. (2004). Proyecto de Resolución del Consejo y de los Representantes de los Estados miembros reunidos en el seno del Consejo, sobre el fortalecimiento de las políticas, sistemas y prácticas en materia de orientación permanente en Europa. Diario Oficial de la Unión Europea, 8448/04 EDUC 89 SOC 179, 19-05-2004, 1-10.

Consejo de Europa. (2008). Proyecto de Resolución del Consejo y de los Representantes de los Gobiernos de los Estados miembros, reunidos en Consejo, de 21 de noviembre de 2008, titulado «Incluir mejor la orientación permanente en las estrategias permanentes de educación y formación permanente». Diario Oficial de la Unión Europea, C 319/4, 13-12-2008, 1-4.

Encina, A. (2008). Gestión del conocimiento en la educación universitaria. Población y Desarrollo, 35, 79-93.

Esteve, J. M., Vera, J., y Aznar, P. (1991). Dimensión Sociocultural del trabajo en el marco europeo. En L. Núñez Cubero (Ed.), Educación y Trabajo (págs. 1131). Sevilla: Preu-Spinola.

Fernández-Salinero, C. (2012). Como gestionar el nuevo conocimiento pedagógico. En L. García Aretio (Ed.), Sociedad del conocimiento y educación (págs. 61-66). Madrid: UNED.

García-Álvarez, J. y Fernández-Salinero, C. (2016). La gestión del conocimiento en el acceso al empleo de los graduados universitarios. Implicaciones en la valoración de la formación. Actas del XVI Congreso Nacional y VII Congreso Iberoamericano de Pedagogía: Democracia y Educación en el siglo XXI (pp. 472). Madrid: Sociedad Española de Pedagogía. http://www.congresodepedagogia.com/libro-de-actas/

Martín-González, M. (2013). Las claves de la educación activa: empleabilidad, eficiencia y evaluación. $L a$ Cuestión Universitaria, 8, 5-14.

Passaillaigue, R., y Estrada, V. (2016). La gestión del conocimiento y el aprendizaje organizacional en instituciones de educación superior. GECONTEC: Revista Internacional de Gestión del Conocimiento y la Tecnología, 4(2), 35-43.

Rodríguez Martínez, A. (2012). Orientación profesional por competencias transversales para mejorar la empleabilidad (Tesis Doctoral). Zaragoza: Universidad de Zaragoza. 